Accelerator Department

BROOKHAVEN NATIONAL LABORATORY

Associated Universities, Inc.

Upton, New York

AGS DIVISION TECHNICAL· NOTE:

No. 120

SDOCHASTIC COOLING FOR PEDESTRIANS

L.W. Smith:

June 21,1976

\title{
I. Introduction..
}

This is a summary of some notes I made a couple of years ago in an attempt to get an elementary understanding of stochastic cooling. Recent. results from the ISR, of which I am only vaguely aware, are not included.

vander Meer ${ }^{1}$ and Palmer $^{2}$ have examined the possibility of damping incoherent beam oscillations by êlectronic feedback (stochastic cooling). This circumvention of Liouville's theorem is achieved by observing the mean lateral position of a sample $N$ of the $N_{\text {i }}$ circulating particles $\quad\left(N / N_{T}\right.$ might typically be $\cong 10^{-5}$ ) Approximately one: quarter of a betatron wave- . length downstream, a momentum kick is applied to move the mean transverse momentum toward zero.". After any "'real" coherent oscillations have been. damped the system operates on the sensed statistical fluctuations of the mean beam position of the finite number, $N$, of the particles sampled.

In Section II I review the damping of coherent oscillations and in Section. III the damping of incoherent oscillations. Section IV points out some posible problem areas which need further study.

Notation similar to Palmer.' ${ }^{2}$ ' is used below.

\section{Coherent Damping:}

At some machine azimuth the mean beam transverse position is sensed and at some angle: $\theta$ (in betatron oscillation phase space) downstream a momentum kick; proportional to the sensed mean beam position, is applied to move (in the impulse approximation) the mean"beam momentum toward.zero." Transit time spread ${ }^{2}$ between sensing and correcting stations is ignored here as are the technical problems of getting the correction signal to arrive at the 
correcting station at the proper time with sufficient band-pass.

Figure 1 is a diagram of the'motion of the mean beam position in betatron phase space.. Units of $\mathrm{y}^{\prime}$ are chosen:so that the orbits are circles.

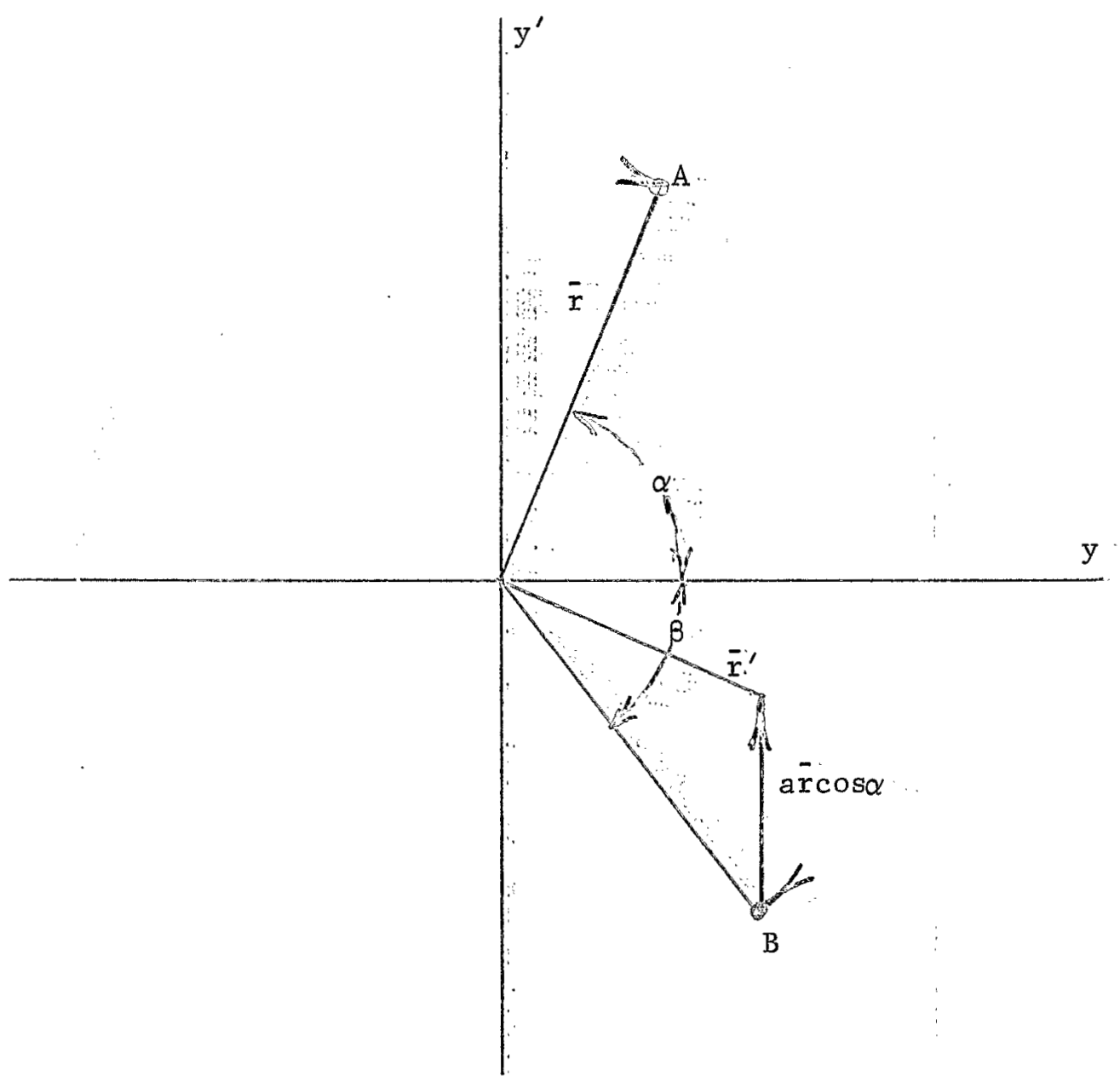

Fig...1

At point A a portion of the beam is within the sensing station and a mean . lateral position, $\bar{r} \cos \alpha$, is detected. At an angle, $\theta=\alpha+\beta$; later a momentum kick, $\delta y^{\prime}$, is given (point B). For the moment:we ignore noise and. image forces. Then $\cdots$

$$
\delta y^{\prime}=a \bar{r} \cdot \cos \alpha
$$


(a"is the "gain" of the feedback system) and :

$$
\bar{r}^{\prime 2}=\bar{r}^{2}+\delta y^{\prime 2}-2 \bar{r} \delta y^{\prime} \sin \beta
$$

Averaging over a11 initia1 phases, $\alpha$;

$$
\left\langle\overline{\mathrm{r}}^{\prime}, 2\right\rangle=\frac{\pi}{2 \pi} \int_{\mathrm{p}}^{2 \pi} \bar{r}^{\prime 2}(\alpha) \mathrm{d} \alpha
$$

we get

$$
\left\langle\bar{r}^{\prime 2}\right\rangle_{\mathrm{p}}=\bar{r}^{2}\left(1-\frac{2 \mathrm{asin} \theta-\mathrm{a}^{2}}{2}\right)
$$

per pass through the system. From (1) we see that damping obtains for

$$
0<a<2 \sin \theta
$$

with optimum ${ }^{3}$ damping for $a=\sin \theta$

Consider now the effect of noise in the system: Define $\left\langle b^{2}\right\rangle^{\frac{7}{2}}$ as the sensing noise in units of the RMS position of one particle. Palmer quotes the ISR value $\left\langle\mathrm{b}^{2}\right\rangle^{\frac{1}{2}}=10^{4} \mathrm{~cm}$, i. e. the RMS error in sensing the mean position of $10^{4}$ particles is $1 \mathrm{~cm}$. The expectation value of the noise contribution to $\bar{r}^{\prime 2}$ is then $\frac{\mathrm{a}^{2}\left\langle\mathrm{~b}^{2}\right\rangle}{\mathrm{N}^{2}}$, and

$$
\left\langle\bar{r}^{2}\right\rangle_{p}=\bar{r}^{2}\left(1-\frac{2 a \sin \theta-a^{2}}{2}+\frac{a^{2}\left\langle b^{2}\right\rangle}{N^{2} r^{2}}\right)
$$

and damping obtains for

$$
0<a<\frac{2 \sin \theta}{1+\frac{\left\langle b^{2}\right\rangle}{N^{2}-\dot{r}^{2}}}
$$

If $\left(\bar{r}-\bar{r}^{\prime}\right) / \bar{r}$ per pass through the system is sma11 Eq..(2): can be written (we drop the notation 〈 $\rangle_{\mathrm{p}}$ for simplicity) 


$$
\bar{r}^{\prime} \cong \bar{r}\left(1-\frac{2 a \sin \theta-a^{2}}{4}+\frac{a^{2}\left\langle b^{2}\right\rangle}{2 N^{2}-2}\right),
$$

and if a driving term due, e.g., to image forces exists

$$
\bar{r}^{\prime} \cong \bar{r}\left(1-\frac{2 a \sin \theta-a^{2}}{4}+\frac{a^{2}\left\langle b^{2}\right\rangle}{2 N^{2}-2}+\lambda N\right)
$$

$\lambda$ can be a function of the vacuum chamber parameters, azimuthal extent of the sensing station, etc. Ignored here is any possible beneficial Landau damping due to momentum and spread. From Eq (3) damping obtains for $(\sin \theta \cong 1$ )

$$
\frac{2 \lambda N}{1+\frac{\left\langle b^{2}\right\rangle}{N^{2}-2}} \approx a \approx \frac{2(1-\lambda N)}{1+\frac{\left\langle b^{2}\right\rangle}{N^{2}-2}}
$$

\section{III: Incoherent Damping}

We use van der Meer's method of averaging overal1 combinations of ampli-: tude and phase. It is assumed that there is sufficient transit time spread per revolution so that the samples, $N$, are statistically independent from one revolution to the next.. Consider Fig. 1; at position $B$, in phase space; a momentum kick, $\delta y^{\prime}$, proportional to the $y$ coordinate of the center of gravity at point $A$, is given to the beam center of gravity. The $y, y^{\prime}$ coordinates of the center of gravity at point $B$ are; $\ldots$.

$$
\frac{1}{N}\langle\Sigma r \cos \beta\rangle, \frac{1}{N}\langle\operatorname{srsin} \beta\rangle
$$

where the summations are over al 1 particles. After the kick the new coordinates of the i-th particle are $\left([y] ;, \ldots\left[\ddot{y}^{\prime}:\right]\right)$,

$$
\left[r_{i} \cos \beta_{i}\right],\left[r_{i} \sin \beta_{i}-\operatorname{acos} \theta \frac{1}{N}\langle\Sigma r \cos \beta\rangle-a \sin \theta \frac{1}{N}\langle\Sigma r \sin \beta\rangle\right]
$$

We take the square of the new amplitude; sum over $i$ and divide by $N .4$ Then 
integrating over a 11 possible combinations of r and $\bar{\beta}$ to evaluate $\langle\operatorname{rcos} \beta\rangle^{2}$ and $\langle\operatorname{srin} \beta\rangle^{2}$ we get (cross terms do not contribute)

$$
\sigma^{\prime 2}=\sigma^{2}\left(1-\frac{2 a \sin \theta-a^{2}}{2 N}\right)
$$

The functiona 1 similarity to Eq. (1) suggests that this result could have been arrived at more, simply. Indeed it appears that we can, for predicting. average values, use the Ergodic theorem ${ }^{6}$ to tobtain Eq. (4) directly from $\mathrm{Eq}$ : (1).

If we include noise; set $\theta \cong \pi / 2$, and assume a is sma 11 ,

$$
\sigma^{\prime 2}=\sigma^{2}\left(1-\frac{a}{N}+\frac{a^{2}\left\langle b^{2}\right\rangle}{N^{2} \sigma^{2}}\right)
$$

and the system damps for

$$
0<a \sim \frac{\mathrm{No}^{2}}{\left\langle\mathrm{~b}^{2}\right\rangle}
$$

with an optimum gain

$$
a_{0}=\frac{N \sigma^{2}}{2\left\langle b^{2}\right\rangle}
$$

The optimum damping per passage through the system is

$$
\sigma^{\prime 2}=\sigma^{2}\left(1-\frac{\sigma^{2}}{4\left\langle b^{2}\right\rangle}\right)
$$

or

$$
\sigma^{\prime} \cong \sigma\left(1-\frac{\sigma^{2}}{8\left\langle b^{2}\right\rangle}\right)
$$

giving

$$
\sigma(t) \cong \frac{\sigma(0)}{1+\frac{\sigma_{0}^{2} t}{8\left\langle\mathrm{~b}^{2}\right\rangle}}
$$


with a $1 / 2$ folding time (in revolutions) of $8\left\langle b^{2}\right\rangle / \sigma^{2}(0)$. Note that this rate can be maintained only if $a_{0}$ is programmed to decrease proportiona $11 y$ to $\sigma(t)$. Otherwise $\sigma(t)$ will asymtoticaily approach $\sigma(0) / \sqrt{2}$.

\section{Possible Problems}

We have made a number of simplifying assumptions, among them:

a) A smooth distribution in phase space. If there is any "lumpiness" in the phase space density due, e.g., to nearness to a nonlinear resonance, and if the sensing and correcting stations have sufficient higher moment sensitivity, antidamping of beam envelope oscillations could occur. Also, if the distribution in phase space is non-smooth the evaluation of $\langle\Sigma r \cos \theta\rangle^{2}$ and $\langle\operatorname{Lrsin} \beta\rangle^{2}$ is (to me) obscure. This also implies a non-stationary process, i.e. a dependence on the absolute origin in time so the Ergodic theorem could not: be applied.

b) "Only the mean square noise has been considered. In practice b would probably be distributed similarly to the Rayleigh distribution with a probability density function

$$
P(b) \cong \frac{2 b}{\left\langle b^{2}\right\rangle} \exp \left(-b^{2} /\left\langle b^{2}\right\rangle\right)
$$

and one might expect this to lead to a long tailed distribution in particle: position... However since the noise contributions to $y^{\prime \prime}$ (and $y$ ) are sma 11 and in large number, the Central Limit theorem would predict that the distribution in $\mathrm{y}^{\prime \prime}$ (and $\mathrm{y}$ ) approaches the Normal distribution. If the beam has some ini-. tial $\mathrm{y}_{\max }$ (due, e.g., to shaving) any noise wi11 populate the region $\mathrm{y}_{>} \mathrm{y}_{\max }{ }^{\bullet}$

c) Only damping in two dimensional phase space has been considered: If there is mixing in six dimensional phase space, complications could result.:

d) Bandwidth considerations have been ignored. To damp incoherent oscillations a large bandwidth is required... As suming that the bandwidth (and noise) of the system is set by the RC of the sensing station, deterioration of the signal to noise ratio will start ${ }^{7}$ at frequencies $f \widetilde{\gtrless}(2 \pi R C)^{-1}$," which is just the frequency needed to "instantaneously" sample the fluctuations of the $N$ particles within the station.: Since $C \approx N$ (i.e.e. the length of ,the station) one may reduce the bandwidth at the expense of damping time but the deterioration of the signal to noise ratio will remain. 


\section{Conclüsions}

For an "idea1" system"stochastic cooling seems to work; but technica1 difficulties may limit its usefulness. Computer simulations of "non-idea 1 ". systems could be informative if round-off errors, etc. can be kept sma11: enough.

\section{References}

1. S. van der Meer, CERN/ISR-P0/72-13. (CRISP CERN/ 7:-31).

2.: R.B...Palmer; CRISP 73-19.(BNL 18395).

3. Physicaliy, this is due to the fact that in the absence of any phase information the best bet is $\alpha=0$ since the probability density distribution of $y$ is $\frac{1}{\pi\left(r^{2}-y^{2}\right)^{\frac{1}{2}}}$.

4. … Actually $\frac{1}{N} \Sigma r_{i}^{2}$ is a biased estimator of $\sigma \%$ but since $N \gg 1$ the point is academic.

5.. Using Parseva1' s' theorem, see:e.g., E.T. Whittaker and G.N. Watson, "Modern :Analysis", Cambridge, N.Y.

6. Roughly, the Ergodic theorem:states that averages performed in time on $\ldots$ a typical member of a system give the same results as corresponding averages taken over the, system at the same moment; i.e. time and ensemble averages are equivalent under suitable conditions. See e.g. J.I. . Doob;.." Stochastic Processes", Wiley, N.Y.

7. Another way of looking at this is that the impulse approximation breaks down when the particle transit time through the station approaches the system rise time.

LWS/ 1 sk

Distr: Dept.:Admin. AGS Division S\&P

Scientific Staff in EP\&S, ISA, and PD 